\title{
Ethnicity and Neonatal Lupus Erythematosus Manifestations Risk in a Large Multiethnic Cohort
}

\author{
Talia Diaz ${ }^{1}$ (D), Daniela Dominguez ${ }^{1}$ (D), Edgar Jaeggi², Andrea M. Knight ${ }^{3}$ (D), Carl A. Laskin ${ }^{4}$ (D), \\ Lawrence $\mathrm{Ng}^{1}$, Franklin Silverio ${ }^{1}$, Earl D. Silverman ${ }^{5}$, and Linda T. Hiraki ${ }^{6}$
}

\begin{abstract}
Objective. To evaluate the association between ethnicity and neonatal lupus erythematosus (NLE), as well as specific NLE manifestations in a large multiethnic population.

Methods. We conducted a cohort study of the children ( $\leq 1 \mathrm{yr}$ of age) seen in the NLE clinic at The Hospital for Sick Children (SickKids), between January 2011 and April 2019. The cohort was divided into European, non-European, and mixed European-non-European groups according to parent-reported child's ethnicity (Canada Census categories). Outcomes were NLE and specific NLE manifestations (cardiac, cutaneous, cytopenias, transaminitis, and macrocephaly). The frequency of NLE and specific manifestations were compared between ethnic groups (Fisher exact test). We tested the association between ethnicity and (1) NLE risk, and (2) specific NLE manifestations with logistic regression models, including covariates for child's sex, maternal rheumatic disease status during pregnancy, and maternal use of antimalarials during pregnancy (multiple comparisons threshold $P<0.008$ ).

Results. We included 324 children born to 270 anti-Ro antibody-positive mothers. Median age at first visit was 1.8 (IQR 1.4-2.3) months, and median follow-up time was 12 (IQR 2-24) months. The majority was non-European (48\%), with 34\% European, and 18\% mixed European-non-European. There was no significant association between non-European ethnicity (OR 1.18, 95\% CI 0.71-1.94, $P=0.51$ ), mixed European-non-European ethnicity (OR 1.13, 95\% CI 0.59-2.16, $P=0.70$ ), and NLE risk compared with European ethnicity. We also did not find an association between ethnicity and specific NLE manifestations in univariate or multivariable-adjusted models.

Conclusion. In a large multiethnic cohort, there was no association between a child's ethnicity and NLE risk or specific NLE manifestations.
\end{abstract}

Key Indexing Terms: autoantibodies, cohort studies, congenital heart block, pediatric rheumatic diseases, pregnancy, risk factors

LTH is supported by a grant from the Arthritis Society (TAS; Grant ID\# 18-0259, Stars Career Development Award).

${ }^{I}$ T. Diaz, $M D$, D. Dominguez, $M D, M S c, L . N g, B S c$, F. Silverio, BSc, Division of Rheumatology, The Hospital for Sick Children;

${ }^{2}$ E. Jaeggi, MD, Fetal Cardiac Program, The Hospital for Sick Children, and Department of Paediatrics, University of Toronto; ${ }^{3}$ A.M. Knight, MD, MSCE, Division of Rheumatology, Neurosciences and Mental Health Program, Research Institute, The Hospital for Sick Children, and Department of Paediatrics, University of Toronto; ${ }^{4}$ C.A. Laskin, MD, Mount Sinai Hospital, University of Toronto; ${ }^{5}$ E.D. Silverman, MD, FRCPC, Division of Rheumatology, and Translational Medicine, Research Institute, The Hospital for Sick Children, and Department of Paediatrics, University of Toronto; ${ }^{6}$ L.T. Hiraki, MD, FRCPC, ScD, Division of Rheumatology, The Hospital for Sick Children, Genetics and Genome Biology, Research Institute, The Hospital for Sick Children, and Department of Paediatrics, University of Toronto, Toronto, Ontario, Canada.

The authors declare no conflicts of interest.

Address correspondence to Dr. L.T. Hiraki, Research Institute, The Hospital for Sick Children, PGCRL, 686 Bay Street, Toronto, ON M5G 0A4, Canada.Email: linda.hiraki@sickkids.ca.

Accepted for publication March 19, 2021.
Neonatal lupus erythematosus (NLE) is a passively acquired autoimmune disorder secondary to the transplacental passage of maternal anti-Ro antibodies. ${ }^{1}$ These antibodies are present in $90 \%$ of women with Sjögren syndrome (SS) and in $20-30 \%$ of women with systemic lupus erythematosus (SLE).

Despite the high prevalence of anti-Ro antibodies in women with rheumatic diseases, $50 \%$ of children with NLE are born to asymptomatic women $(25 \%)$ or women with an undifferentiated autoimmune syndrome $(25 \%){ }^{2}$

NLE is characterized by cardiac and noncardiac manifestations. Cardiac NLE may manifest as conduction tissue disease (congenital heart block, sinus bradycardia), endomyocardial disease (myocarditis, dilated cardiomyopathy, valvular abnormalities, and endocardial fibroelastosis), or both. ${ }^{3}$ The noncardiac NLE manifestations are cutaneous rash, elevated liver enzymes, cytopenias, and benign macrocephaly. ${ }^{4}$ The noncardiac manifestations, except benign macrocephaly, are usually transient and spontaneously resolve with clearance of maternal anti-Ro antibodies.

The most severe NLE manifestation is congenital complete heart block $(\mathrm{CHB})$, which is irreversible. NLE is the most common cause of $\mathrm{CHB}$, accounting for $>90 \%$ of perinatal cases. ${ }^{5} \mathrm{CHB}$ is present in $1-5 \%$ of children born to women with 
high-titer anti-Ro antibodies. The risk of $\mathrm{CHB}$ increases to up to $18 \%$ for children born to women with a previous child with cardiac NLE. ${ }^{1}$

Although the presence of maternal anti-Ro antibodies is necessary for NLE, ${ }^{6}$ it is not the sole risk factor causing NLE, since the majority of children born to anti-Ro antibody-positive mothers are unaffected. The risk of NLE is likely influenced by other factors such as anti-Ro antibody titers ${ }^{6}$ and maternal rheumatic disease status during pregnancy. ${ }^{7}$ It has also been demonstrated that maternal antimalarial use during pregnancy reduces the risk of cardiac NLE as well as cutaneous NLE. $8,9,10,11$

Previous studies have suggested that ethnicity may also influence NLE outcomes, with more frequent adverse cardiac NLE outcomes reported among children of non-European ethnicity compared to children of European ethnicity. ${ }^{7,12}$ No study to date has examined the association between ethnicity and noncardiac NLE manifestations. The aim of this study was to examine the association between ethnicity and NLE, as well as specific NLE manifestations within a large, multiethnic, single-center cohort.

\section{METHODS}

Study population. We conducted a cohort study of all patients followed in our NLE clinic at The Hospital for Sick Children (SickKids), born to women with positive anti-Ro antibodies and seen at $\leq 1$ year of age, between January 2011 and April 2019. The clinic serves a multiethnic population in the greater Toronto area. Mothers eligible for the study were those with positive anti-Ro antibodies as part of a rheumatic disease diagnosis, a maternal history of symptoms prompting autoantibody testing, and/ or a delivery of a prior child with NLE. The anti-Ro antibody status of all mothers was confirmed in the first trimester of pregnancy. Serial fetal echocardiograms were performed beginning at 16-18 weeks gestational age and throughout the pregnancy at intervals determined by antibody titer and echocardiogram findings. ${ }^{12}$ Postnatally, children born to these mothers were seen in the NLE clinic at 2, 4, and if necessary, 12 months of age, irrespective of the presence or absence of NLE manifestations. At the first NLE clinic visit, we complete electrocardiograms on all infants, along with complete blood counts, liver function tests, and autoantibody tests. If abnormal, these studies are repeated until normalized. At each visit, weight, length, and head circumference are measured. A head circumference increasing disproportionately faster than weight and length prompts further investigations, such as a head ultrasound. Clinical and laboratory manifestations of NLE are prospectively collected and stored in a dedicated NLE database. Beginning in 2011, families were routinely asked to report the ethnicity of mother and child.

Study participants were excluded if missing any of the following: (1) documentation of positive anti-Ro antibodies during pregnancy; (2) self-reported ethnicity for mother and child; or (3) documentation of maternal medication use during pregnancy.

We extracted demographic data on mothers and children from the NLE database and medical charts. Self-reported ethnicity was collected by questionnaire, using Canada Census categories. Participants were grouped based on child's ethnicity into categories: European, East Asian, South Asian, African, Latin American, and Mixed ( $\geq 2$ ethnicities). Subsequently, we stratified the cohort into 3 groups: European, non-European, and mixed European-non-European.

Our outcome, NLE diagnosis, was defined as the presence of $\geq 1$ of the following manifestations: (1) Cardiac: conduction (congenital heart block, sinus bradycardia) and/or endomyocardial disease (myocarditis, dilated cardiomyopathy, valvular abnormalities, and endocardial fibroelastosis); (2) Cutaneous: NLE rash; (3) Hepatic: elevated liver enzymes (level above the age-appropriate parameter); (4) Hematologic: hemolytic anemia, neutropenia and/or thrombocytopenia (level below the age-appropriate parameter); and (5) Neurologic: benign macrocephaly confirmed by head ultrasound with findings consistent with NLE (extraaxial fluid and normal ventricular sizes). We also studied each of the NLE manifestations individually. In order to ensure complete capture of transient NLE manifestations, we defined requirements for the age at first visit for NLE rash at 2-4 months of age, cytopenias and transaminitis at $\leq 2$ months of age, benign macrocephaly at 4-12 months of age, and CHB at any time prenatally or $\leq 1$ year of age.

We reviewed self-reported maternal rheumatic disease status and antimalarial use during pregnancy. Antimalarial (hydroxychloroquine [HCQ] or chloroquine) use during pregnancy was defined as medication use before pregnancy or started during the first 2 weeks of pregnancy, and continued throughout. We also reviewed the prevalence of anti-La antibodies in our study cohort.

Demographic, clinical, and laboratory data were analyzed using $t$ tests and Fisher exact tests where appropriate. We tested the association between ethnicity and NLE, as well as specific NLE manifestations, in logistic models. Multivariable models included covariates for child's sex, maternal rheumatic disease status during pregnancy (present/absent), and maternal use of antimalarials during pregnancy (taken/not taken).

Sensitivity analyses were restricted to the first-born child. We also tested the association between maternal ethnicity and NLE risk and NLE manifestations, including history of a prior child with NLE as covariate, and added anti-La antibody status in multivariable models. A Bonferroni corrected $P$ value $<0.008$ (adjust for 6 independent tests) was used for significance. Data analysis was performed using R version 1.1.456 (R Foundation for Statistical Computing). This study was approved by the research ethics board at SickKids (REB no. 1000034004).

\section{RESULTS}

A total of 339 children were screened for our study. Of those, 15 were excluded due to negative maternal anti-Ro antibodies during the pregnancy, a first NLE clinic visit $>1$ year of age, unavailable ethnicity data, or unconfirmed status of antimalarial use during pregnancy. The study cohort included 324 children, with $51 \%$ female ( $\mathrm{n}=165$; Table 1$)$. Of the 324 children, $14 \%$ ( $n=46$ ) were second- or third-born children, and of those, $21 \mathrm{had}$ an older sibling with NLE (6.5\% of total cohort). The median age at first visit was 1.8 months (IQR 1.4-2.3 months) and the majority of children had their first visit at $\leq 6$ months of age $(98 \%, n=318)$. Children were followed for a median duration of 12 months (IQR 2-24 months). Thirty-six percent ( $\mathrm{n}=117$ ) of the children were not seen at 1 year of age. Participants had their first visit within the relevant time window for each NLE manifestation.

The ethnic distribution was 34\% European $(n=110), 48 \%$ non-European $(n=156)$, and $18 \%$ mixedEuropean-non-European $(\mathrm{n}=58)$ ethnicity. The most prevalent non-European ethnicities were East Asian (31\%) and South Asian (27\%), followed by African (21\%), Latin American (5\%), and mixed non-European ancestry (16\%). There were no differences in the age at first visit between ethnic groups (European 2.2 [SD 1.4] months; non-European 2.3 [1.8] months; mixed European-non-European 2.3 [SD 1.4] months, $P=0.44$ ), or age at last follow-up visit (European 16.9 [SD 16.3] months; non-European 17.1 [16.1] months; mixed European-non-European 17.2 [SD 15.3] months, $P=0.91$ ).

Over half of the children in our cohort had NLE $(54 \%$, 
Table 1. Child demographic and NLE manifestations $(\mathrm{n}=324)$.

n $(\%)$

\begin{tabular}{lc}
\hline Female sex & $165(51)$ \\
Ethnicity & \\
European $^{\text {Non-European }}{ }^{\mathrm{a}}$ & $110(34)$ \\
Mixed European-non-European & $156(48)$ \\
NLE & $58(18)$ \\
Cardiac & $175(54)$ \\
1st degree CHB & $23(13)$ \\
2nd degree CHB & $2(9)$ \\
3rd degree CHB & $2(9)$ \\
Endocardial fibroelastosis & $14(61)$ \\
Myocarditis & $7(30)$ \\
Hepatic & $5(22)$ \\
Hematologic & $92(53)$ \\
Neutropenia & $69(39)$ \\
Thrombocytopenia & $54(78)^{\mathrm{c}}$ \\
Neutropenia and thrombocytopenia & $11(16)^{\mathrm{c}}$ \\
Cutaneous & $4(6)^{\mathrm{c}}$ \\
Neurologic (benign macrocephaly) & $37(21)$ \\
\end{tabular}

Data presented as n (\%). Median values for neutropenia $0.78 \times 10^{9} / \mathrm{L}$ (range 0.26-0.99), thrombocytopenia $98 \times 10^{\circ} / \mathrm{L}$ (range 63-186), ALT $71 \mathrm{U} / \mathrm{L}$ (range 52-314), AST $97 \mathrm{U} / \mathrm{L}$ (range 77-280). ${ }^{a}$ Non-European group comprised patients of East Asian $(n=49,31 \%)$, South Asian $(\mathrm{n}=42,27 \%)$, African $(\mathrm{n}=32,21 \%)$, Latin American $(\mathrm{n}=8,5 \%)$, and mixed Non-European $(\mathrm{n}=25,16 \%)$ ethnicity. ${ }^{\mathrm{b}}$ Age-adjusted normal values were used. 'Shown as percentage of patients with hematologic involvement and not of total cohort. ALT: alanine aminotransferase, AST: aspartate aminotransferase; CHB: complete heart block; NLE: neonatal lupus erythematosus.

$\mathrm{n}=175)$. Of these, $13 \%(\mathrm{n}=23)$ had cardiac involvement, $61 \%$ of whom had $3 \mathrm{rd}$ degree CHB $(\mathrm{n}=14)$. The most common NLE manifestation was hepatitis ( $53 \%, \mathrm{n}=92$; Table 1$)$.

Our study included 270 mothers, with 196 mothers (73\%) having a rheumatic disease diagnosis during pregnancy. The most frequent diagnoses were SLE in 66\% $(n=129 / 196)$ and SS in $18 \%(n=36 / 196)$. Of the mothers with rheumatic disease, $52 \%(n=101 / 196)$ reported taking antimalarials during pregnancy compared to $1 \%(n=1 / 74)$ of women without rheumatic disease diagnoses. As expected, a higher proportion of mothers with SLE were on antimalarials during pregnancy compared to mothers with other rheumatic diseases (66\% vs $26 \%, P<0.0001)$. The prevalence of anti-La antibody positivity during pregnancy was $26 \%(\mathrm{n}=84)$, anti-La antibody negativity was $41 \%(\mathrm{n}=132)$, and $33 \%(\mathrm{n}=108)$ were missing anti-La antibody status.

We found no significant difference in NLE risk between non-European (OR 1.18, 95\% CI 0.71-1.94, $P=0.51$ ) and mixed European-non-European children (OR 1.13, 95\% CI 0.59-2.16, $P=0.70)$, compared with European children (Table 2). We also did not find an association between ethnic group and cardiac NLE (non-European: OR 0.53, 95\% CI 0.19-1.43, $P=0.21$; and mixed European-non-European: OR 0.91 , 95\% CI $0.28-2.89, P=0.88$ ), nor cutaneous NLE (non-European: OR 1.48, 95\% CI 0.67-3.25, $P=0.32$; and mixed European-non-European: OR 0.87, 95\% CI 0.28-2.67, $P=0.81$; Table 2, Figure 1). This held true in univariate and multivariable-adjusted analyses.

Analyses restricted to the first-born child resulted in a sample size of 260 children, with no significant change in effect of non-European ethnicity on the odds of NLE (OR 1.20, 95\% CI $0.69-2.10, P=0.51$ ), or mixed European-non-European (OR 0.89, 95\% CI $0.42-1.86, P=0.76)$ compared with European children on specific NLE manifestations. Adding anti-La antibody status to multivariable models did not significantly change the association between ethnicity and NLE or NLE manifestations (Supplementary Table 1, available with the online version of this article).

Sensitivity analyses testing the association of maternal ethnicity, instead of child's ethnicity, also did not demonstrate a statistically significant association with NLE risk nor specific NLE manifestations. Also, a sensitivity analysis testing the association between children's ethnicity and NLE risk and specific NLE manifestations including history of a prior child with NLE as covariate, did not demonstrate a statistically significant association (data not shown).

\section{DISCUSSION}

In our multiethnic population of children born to anti-Ro antibody-positive mothers, we did not observe a statistically significant association between a child's ethnicity and NLE. There was no difference in the odds of NLE nor specific NLE manifestations between children of non-European and mixed European-non-European ancestry, compared with European ancestry, with and without accounting for child's sex, maternal rheumatic disease status, and antimalarial use during pregnancy.

The prevalence of NLE manifestations in our population was similar to that reported in the literature, with the exception of hepatic involvement. A total of 53\% of the babies we assessed had evidence of NLE transaminitis. The prevalence of NLE transaminitis reported in the literature is approximately $9-25 \% .{ }^{13}$ Our higher prevalence is likely due to our clinical practice of routine screening of all infants born to anti-Ro-positive mothers for hepatic involvement. The prevalence of NLE transaminitis we observed in our population is similar to other prospective studies that routinely monitored liver function in infants approximately 3 months of age, born to anti-Ro antibody-positive mothers. ${ }^{14,15}$ In our population, other NLE manifestations such as rash and cytopenias were found with the same frequency as those reported in prior studies. ${ }^{1,16}$ Although neutropenia was the most common hematologic manifestation of NLE (78\%), we did not observe any cases of neonatal sepsis in these infants. Thrombocytopenia secondary to NLE usually develops within the first week of life. ${ }^{4}$ Since the first NLE clinic visit is between $4-8$ weeks of age, our data may underestimate the prevalence of thrombocytopenia. We found that $7 \%$ of infants had benign macrocephaly associated with NLE, which is consistent with the frequency reported in the literature of $8-11 \%{ }^{4}$

Previous studies report CHB affecting 2\% of first-born children of women with positive anti-Ro antibodies; this percentage increases to $15-18 \%$ in children born to women with a previous 
Table 2. Predictors of NLE risk and specific manifestations $(\mathrm{n}=324)$.

\begin{tabular}{|c|c|c|c|c|c|c|}
\hline Covariate & NLE & Cardiac & $\begin{array}{l}\text { Cutaneous } \\
\text { OR }(95 \% \mathrm{CI}), P\end{array}$ & Hematologic & Hepatic & Neurologic \\
\hline \multicolumn{7}{|l|}{$\begin{array}{l}\text { Child ethnicity } \\
\text { (ref: European) }\end{array}$} \\
\hline Non-European & $1.18(0.71-1.94), 0.51$ & $0.53(0.19-1.43), 0.21$ & $1.48(0.67-3.25), 0.32$ & $1.40(0.76-2.60), 0.27$ & $1.04(0.59-1.84), 0.86$ & $0.36(0.10-1.31), 0.12$ \\
\hline non-European & $1.13(0.59-2.16), 0.70$ & $0.91(0.28-2.89), 0.88$ & $0.87(0.28-2.67), 0.81$ & $0.85(0.36-1.98), 0.70$ & $1.39(0.69-2.81), 0.34$ & $0.54(0.10-2.79), 0.47$ \\
\hline Male sex (ref: female) & $0.71(0.46-1.11), 0.14$ & $0.52(0.21-1.29), 0.16$ & $0.98(0.49-1.95), 0.95$ & $0.63(0.36-1.09), 0.10$ & $0.54(0.33-0.90), 0.01$ & 2.35 (0.70-7.88), 0.16 \\
\hline $\begin{array}{l}\text { Antimalarials in } \\
\text { pregnancy }\end{array}$ & $0.78(0.46-1.30), 0.34$ & $0.80(0.24-2.67), 0.72$ & $0.68(0.29-1.55), 0.36$ & $0.84(0.46-1.54), 0.57$ & $0.58(0.32-1.05), 0.07$ & $1.46(0.40-5.38), 0.56$ \\
\hline $\begin{array}{l}\text { Maternal rheumatic } \\
\text { disease }\end{array}$ & $0.87(0.50-1.51), 0.63$ & $0.30(0.11-0.83), 0.02$ & $1.00(0.44-2.27), 0.99$ & $2.21(1.07-4.55), 0.03$ & $0.99(0.55-1.80), 0.99$ & $1.10(0.24-4.96), 0.90$ \\
\hline
\end{tabular}

Multivariable models adjusted for child's ethnicity, sex, antimalarial use during pregnancy, and maternal rheumatic disease status. NLE: neonatal lupus erythematosus.

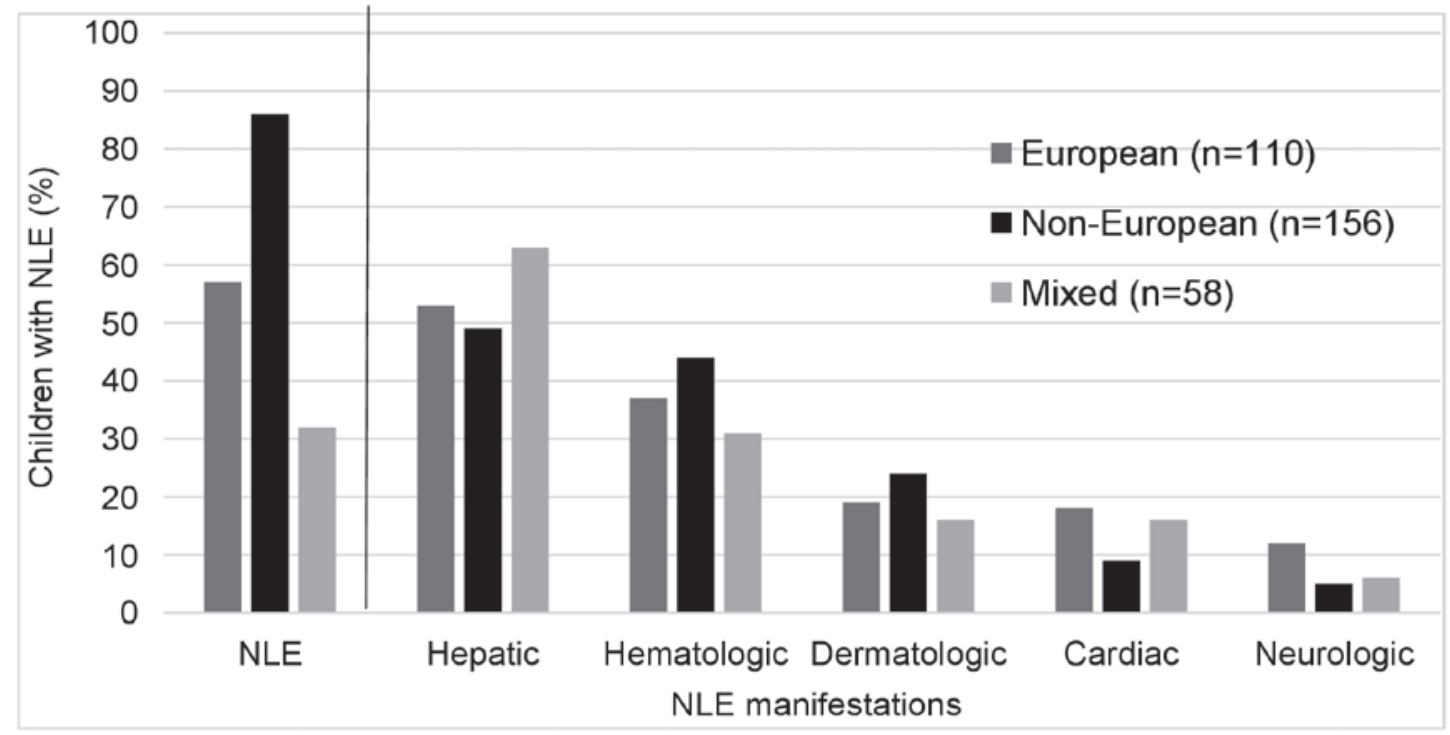

Figure 1. Prevalence of NLE and NLE manifestations stratified by ethnicity. NLE: neonatal lupus erythematosus.

affected child with cardiac NLE. ${ }^{1,3}$ The overall prevalence of 3 rd degree $\mathrm{CHB}$ in our cohort was $4 \%$. This prevalence is consistent with prior prevalence reports since our cohort included not only first-born children but also subsequent children born to women positive for anti-Ro antibodies. In our cohort, 21 children (6\%) had an older sibling with NLE, and of those, 13 (62\%) children had NLE.

Our study findings of no association between ethnicity and NLE are consistent with a multinational study on the effect of HCQ on cardiac NLE risk in 257 pregnancies of anti-Ro antibody-positive mothers from US, UK, or French registries. ${ }^{8}$ In this selected sample of mothers with a previous child with cardiac NLE, there was no association between race/ethnicity and cardiac NLE, after accounting for factors that included HCQ exposure throughout pregnancy (non-White vs White: OR $0.55,95 \% \mathrm{CI} 0.21-1.45, P=0.22$ ). Our study findings are contrary to 1 prior study of ethnicity and cardiac NLE, also from our institution. ${ }^{12}$ This study of 189 anti-Ro-positive mothers referred for fetal echocardiograms in 195 pregnancies identified 9 cases of cardiac NLE. The study found that maternal Asian ethnicity was associated with cardiac NLE (OR 11.5, 95\% CI 1.4-95.1, $P=0.01) .{ }^{14}$ Our differing findings may be due to sampling variation.

Ethnicity represents both genetic and environmental factors that potentially influence NLE risk. Prior genetic studies restricted to single ancestral European and Japanese populations identified HLA alleles for CHB risk, specifically HLA-DRB1 alleles and DQA1, both of which have been previously associated with SLE susceptibility. ${ }^{17,18,19}$ To date, there are no large, multiethnic studies of NLE in children born to anti-Ro antibody-positive mothers; thus, no genetic, ancestral-specific factors have been identified.

The study findings should be considered in light of some limitations. The modest number of patients with cardiac NLE manifestations (13\%) precludes comparisons between non-European ancestral subgroups (i.e., East Asian, South 
Asian, and African ancestries). However modest, our study's non-European sample size is the largest reported to date for studies of NLE. Since our study cohort was drawn from the NLE clinic attended by infants after delivery, we could not examine the association between ethnicity and intrauterine deaths.

Our study had a number of strengths. To our knowledge, this is the first study focused on the association between ethnicity and NLE risk and specific NLE manifestations. The study population is the largest prospective, single-center study of children born to anti-Ro antibody-positive mothers with and without rheumatic disease diagnoses. With the large proportion of anti-Ro antibody-positive mothers without a rheumatic disease, we were able to examine the attributable risk of maternal disease status and medication exposure with NLE. Since our data were prospectively collected from infants undergoing routine screening for NLE manifestations, our reported prevalence of NLE and its specific manifestations likely reflect the true prevalence of NLE in children born to mothers positive for anti-Ro antibodies.

In conclusion, in this large, multiethnic, single-center cohort study of children born to anti-Ro antibody-positive mothers, the child's ethnicity was not associated with NLE risk nor specific NLE manifestations. Further studies in multiethnic cohorts are needed to validate our findings. Future genetic studies will enable the identification of more precise mechanisms influencing NLE risk and manifestations.

\section{ONLINE SUPPLEMENT}

Supplementary material accompanies the online version of this article.

\section{REFERENCES}

1. Cimaz R, Spence DL, Hornberger L, Silverman ED. Incidence and spectrum of neonatal lupus erythematosus: a prospective study of infants born to mothers with anti-Ro autoantibodies. J Pediatr 2003;142:678-83.

2. Vanoni F, Lava SAG, Fossali EF, Cavalli R, Simonetti GD, Bianchetti MG, et al. Neonatal systemic lupus erythematosus syndrome: a comprehensive review. Clin Rev Allergy Immunol 2017;53:469-76.

3. Izmirly P, Saxena A, Buyon JP. Progress in the pathogenesis and treatment of cardiac manifestations of neonatal lupus. Curr Opin Rheumatol 2017;29:467-72.

4. Silverman E, Jaeggi E. Non-cardiac manifestations of neonatal lupus erythematosus. Scand J Immunol 2010;72:223-5.

5. Skog A, Lagnefeldt L, Conner P, Wahren-Herlenius M, Sonesson SE. Outcome in 212 anti-Ro/SSA-positive pregnancies and population-based incidence of congenital heart block. Acta Obstet Gynecol Scand 2016;95:98-105.

6. Jaeggi E, Laskin C, Hamilton R, Kingdom J, Silverman E. The importance of the level of maternal anti-Ro/SSA antibodies as a prognostic marker of the development of cardiac neonatal lupus erythematosus: a prospective study of 186 antibody-exposed fetuses and infants. J Am Coll Cardiol 2010;55:2778-84.
7. Izmirly PM, Saxena A, Kim MY, Wang D, Sahl SK, Llanos C, et al. Maternal and fetal factors associated with mortality and morbidity in a multi-racial/ethnic registry of anti-SSA/Ro-associated cardiac neonatal lupus. Circulation 2011;124:1927-35.

8. Izmirly PM, Costedoat-Chalumeau N, Pisoni CN, Khamashta MA, Kim MY, Saxena A, et al. Maternal use of hydroxychloroquine is associated with a reduced risk of recurrent anti-SSA/Ro-antibodyassociated cardiac manifestations of neonatal lupus. Circulation 2012;126:76-82.

9. Barsalou J, Jaeggi E, Laskin CA, Brown P, Tian SY, Hamilton RM, et al. Prenatal exposure to antimalarials decreases the risk of cardiac but not non-cardiac neonatal lupus: a single-centre cohort study. Rheumatology 2017;56:1552-9.

10. Barsalou J, Costedoat-Chalumeau N, Berhanu A, Fors-Nieves $\mathrm{C}$, Shah U, Brown P, et al. Effect of in utero hydroxychloroquine exposure on the development of cutaneous neonatal lupus erythematosus. Ann Rheum Dis 2018;77:1742-9.

11. Izmirly P, Kim M, Friedman DM, Costedoat-Chalumeau N, Clancy $\mathrm{R}$, Copel JA, et al. Hydroxychloroquine to prevent recurrent congenital heart block in fetuses of anti-SSA/Ro-positive mothers. J Am Coll Cardiol 2020;76:292-302.

12. Kan N, Silverman ED, Kingdom J, Dutil N, Laskin C, Jaeggi E. Serial echocardiography for immune-mediated heart disease in the fetus: results of a risk-based prospective surveillance strategy. Prenat Diagn 2017;37:375-82.

13. Kobayashi R, Mii S, Nakano T, Harada H, Eto H. Neonatal lupus erythematosus in Japan: a review of the literature. Autoimmunity Rev 2009;8:462-6.

14. Wisuthsarewong W, Soongswang J, Chantorn R. Neonatal lupus erythematosus: clinical character, investigation, and outcome. Pediatric Dermatol 2011;28:115-21.

15. Zuppa AA, Riccardi R, Frezza S, Gallini F, Luciano RM, Alighieri G, et al. Neonatal lupus: follow-up in infants with anti-SSA/ Ro antibodies and review of the literature. Autoimmunity Rev 2017; 16:427-32.

16. Neiman AR, Lee LA, Weston WL, Buyon JP. Cutaneous manifestations of neonatal lupus without heart block: characteristics of mothers and children enrolled in a national registry. J Pediatr 2000;137:674-80.

17. Miyagawa S, Fukumoto T, Hashimoto K, Yoshioka A, Shirai T, Shinohara K, et al. Neonatal lupus erythematosus: haplotypic analysis of HLA class II alleles in child/mother pairs. Arthritis Rheum 1997;40:982-3.

18. Clancy RM, Marion MC, Kaufman KM, Ramos PS, Adler A, International Consortium on Systemic Lupus Erythematosus Genetics, et al. Identification of candidate loci at 6p21 and 21q22 in a genome-wide association study of cardiac manifestations of neonatal lupus. Arthritis Rheum 2010;62:3415-24.

19. Meisgen S, Ostberg T, Salomonsson S, Ding B, Eliasson H, Malarstig A, et al. The HLA locus contains novel foetal susceptibility alleles for congenital heart block with significant paternal influence. J Intern Med 2014;275:640-51. 